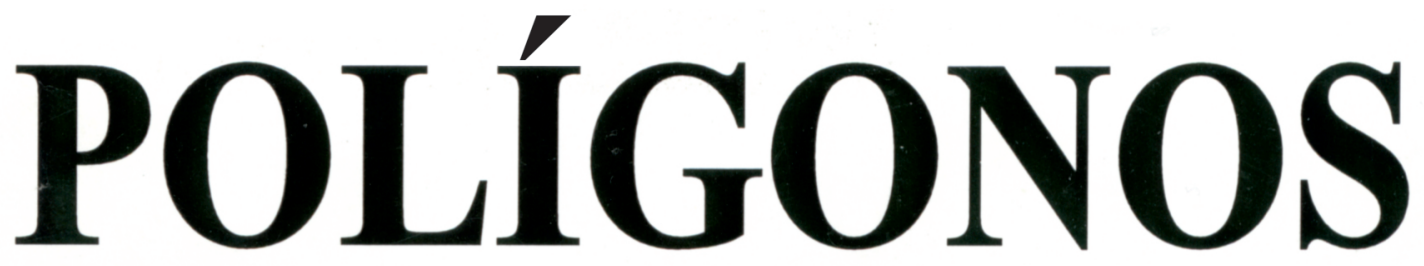

REVISTA DE GEOGRAFÍA

2019

\title{
Segunda época
}

31
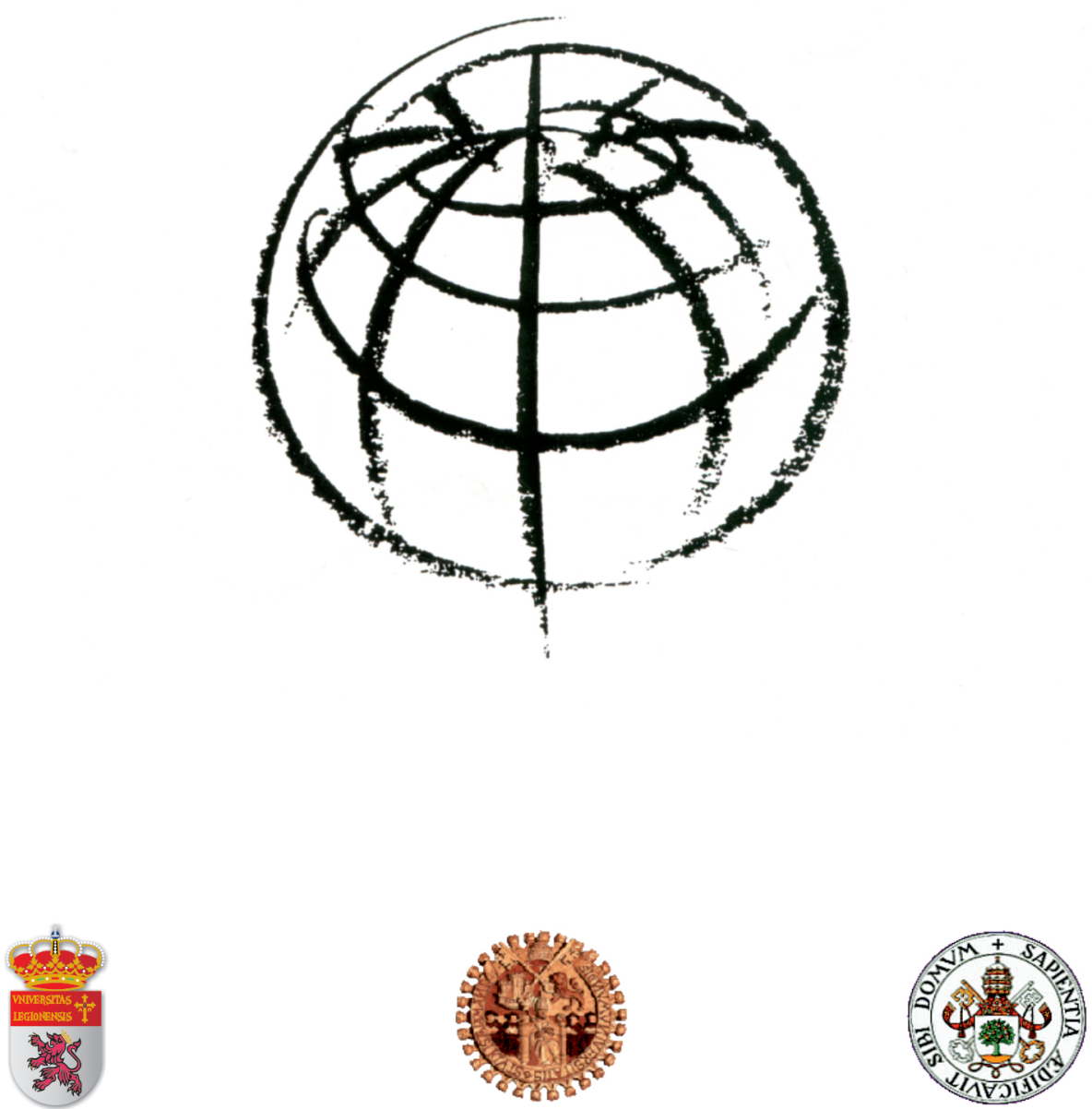

Universidad de León

Departamento de Geografía y Geología
Universidad de Salamanca Departamento de Geografía
Universidad de Valladolid Departamento de Geografía 


\title{
POLÍGONOS
}

Revista de Geografía

\section{CONSEJO DE REDACCIÓN}

\section{DIRECTORES}

Eugenio Baraja Rodríguez (Univ. de Valladolid)

Antonio Ceballos Barbancho (Univ. de Salamanca)

Raquel Domínguez Fernández (Univ. de León)

\section{SECRETARIO}

José Cortizo Álvarez (Univ. de León)

VOCALES

José Luis Alonso Sánchez (Univ. de Salamanca)

Paz Benito del Pozo (Univ. de León)

Carmen Cañizares Ruiz (Univ. de Castilla-La Mancha)

José Luis García Cuesta (Univ. de Valladolid)

Felipe Leco Berrocal (Univ. de Extremadura)

Rafael Mata Olmo (Univ. Autónoma de Madrid)

Carlos G. Morales Rodríguez (Univ. de Valladolid)

David Ramos Pérez (Univ. de Salamanca)

Juan Sevilla Álvarez (Univ. de Oviedo)

\section{CONSEJO ASESOR}

Dr. Valentín Cabero Diéguez. Universidad de Salamanca

Dra. Carminda Cavaco. Universidad de Lisboa

Dr. Pablo José Cicolella. Universidad de Buenos Aires

Dr. Pedro Cunill Grau. Universidad Central de Venezuela Dr. Michael Drain. C.N.R.S. Marsella

Dr. Jorge Gaspar. Universidad de Lisboa

Dr. André Humbert. Universidad de Lorena

Dr. Javier Martín Vide. Universidad de Barcelona

Dr. Eduardo Martínez de Pisón. Universidad Autónoma de Madrid Dr. Ricardo Méndez Gutiérrez-del Valle. I.E.G. - CSIC, Madrid
Dra. María del Carmen Ocaña. Universidad de Málaga

Dr. Patrick O'Flanaghan. National University of Ireland Cork

Dr. Jorge Olcina Cantos. Universidad de Alicante

Dr. José Ortega Valcárcel. Universidad de Valladolid

Dr. Francisco Quirós Linarest. Universidad de Oviedo

Dr. Luciano Lourenço. Universidad de Coimbra

Dr. Hugo Romero Aravena. Universidad de Chile Dra. Edith Rosario Jiménez. Univ. de Guadalajara (México)

Dr. Joan-Eugeni Sánchez. Universidad de Barcelona

Dr. Miguel Ângel Troitiño Vinuesa. Univ. Complutense de Madrid

Polígonos está referenciada en los siguientes índices, catálogos y bases de datos:

\section{Latindex}

ISOC

Dialnet

DICE

\author{
REDACCIÓN Y ADMINISTRACIÓN \\ Departamento de Geografía y Geología \\ Facultad de Filosofía y Letras \\ Campus de Vegazana \\ Universidad de León \\ 24071 LEÓN
}

Teléfonos: 987/291043-291044

Fax: 987/291877

Normas para los autores / resúmenes de números anteriores:

http://http://revpubli.unileon.es/index.php/poligonos/about/submissions\#authorGuidelines

La responsabilidad de las opiniones y los hechos consignados en cada artículo, nota o reseña corresponden exclusivamente a los autores. Los editores no se responsabilizan, en ningún caso, de la credibilidad y autenticidad de los trabajos. En toda reproducción de los materiales publicados, total o parcial, es necesario citar su procedencia

ISSN: $1132-1202$

ISSNe - 2444-0272

Depósito legal: LE-539-1992

Editado en León

Editado por los Departamentos de Geografía de las Universidades de León, Salamanca y Valladolid

(C) Del texto: los autores

(C) De la edición: Universidades de León, Salamanca y Valladolid 


\title{
POLÍGONOS
}

\section{Revista de Geografía}

\author{
$2019 n^{0} 31$ \\ ISSN - 1132-1202 \\ ISSNe - 2444-0272
}

Departamento de Geografía y Geología. Universidad de León Departamento de Geografía. Universidad de Salamanca Departamento de Geografía. Universidad de Valladolid 


\section{ÍNDICE}

\section{ARTÍCULOS}

Lorenzo LóPez TrigaL: Elogio y petición del Prof. Dr. Lorenzo López

Trigal, padrino del candidato Excmo. Sr. D. Horacio Capel Sáez. Univer-

sidad de León, 18 de diciembre de 2018.

Horacio CAPEL SÁEz: Discurso de recepción del Doctorado Honoris

Causa por la Universidad de León. Los geógrafos leoneses y la ciudad de

León como arquetipo urbano. Universidad de León, 18 de diciembre de 2018.

María ZúÑIGA y Jorge OLCINA: Cultura del territorio

José Cortizo Álvarez: Premio Nueva Cultura del Territorio, modalidad de

Divulgación. Presentación de Julio Llamazares. Madrid, 30 de mayo de 2019.

Julio Llamazares: Geografia literaria

Juan NoguÉ: Premio Nueva Cultura del Territorio, modalidad de Gestión.

Presentación del Observatorio del Paisaje de Cataluña. Madrid, 30 de mayo de 2019.

Pere Sala i Martí: Premio Nueva Cultura del Territorio, modalidad de Gestión. Madrid, 30 de mayo de 2019.

\section{RESEÑAS BIBLIOGRÁFICAS}

Horacio CAPel SÁEz (2019): Azares y decisiones. Recuerdos personales. Aranjuez, Ediciones Doce Calles, 333 pp. ISBN 978-84-9774-252-3 
Sergio C. Fanjul (2019): La ciudad infinita. Barcelona, Reservoir Books, 192 pp. ISBN 978-84-1751-163-0.

C. K. Ballantyne (2018): Periglacial Geomorphology. Hoboken, John Wiley \& sons, 472 pp. ISBN 978-14-0510-006-9 


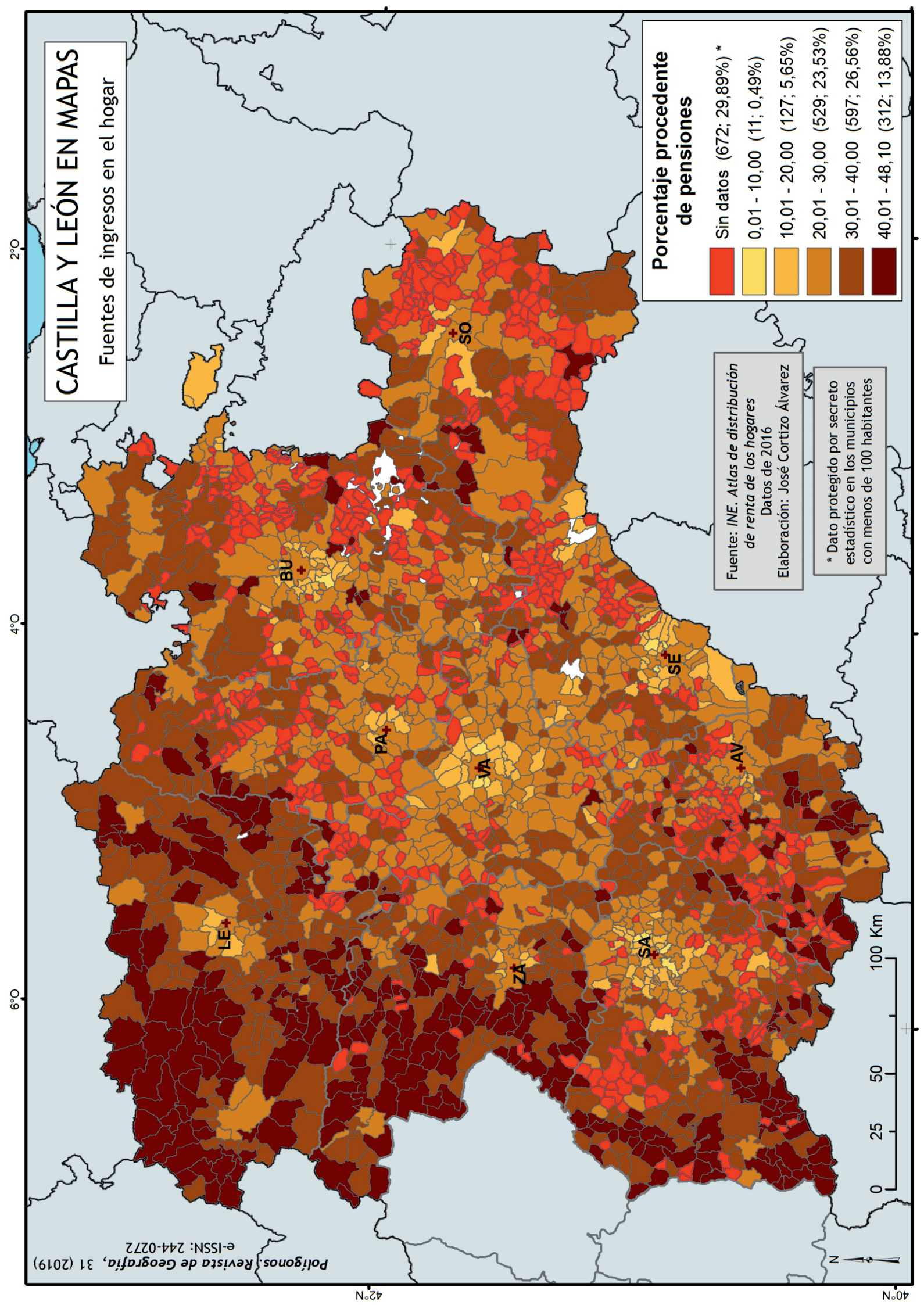




\section{POLÍGONOS}

REVISTA DE GEOGRAFIA

2019 Segunda época

31

5 Consejo de Redacción

Presentación

ARTÍCULOS

11 Lorenzo LÓPEZ TRIGAL

Elogio y petición del Prof. Dr. D. Lorenzo López Trigal, padrino del candidato Excmo. Sr.

D. Horacio Capel Sáez. Universidad de León, 18 de diciembre de 2018

17 Horacio CAPEL

Discurso en la recepción del Doctorado Honoris Causa por la Universidad de León.

Los geógrafos leoneses y la ciudad de León como arquetipo urbano. Universidad de León, 18 de diciembre de 2019

37 María ZÚÑ̃IGA y Jorge OLCINA

Cultura del territorio

45 José CORTIZO ÁLVAREZ

Premio Nueva Cultura del Territorio, modalidad de Divulgación Presentación de Julio Llamazares. Madrid, 30 de mayo de 2019

53 Julio LLAMAZARES

Geografía literaria

57 Juan NOGUÉ

Premio Nueva Cultura del Territorio, modalidad de Gestión. Presentación del Observatorio del Paisaje de Cataluña. Madrid, 30 de mayo de 2019

61 Pere SALAiMARTÍ

Premio Nueva Cultura del Territorio, modalidad de Gestión. Madrid, 30 de mayo de 2019

67 RESEÑAS BIBLIOGRÁFICAS 